\title{
Do Recent Stochastic Tools Help To Better Understand Investors' Preference And Asset Allocation?
}

\author{
Maria-Lenuţa Ciupac-Ulici, Commercial Academy Foundation, Romania \\ Mircea-Cristian Gherman, Technical University of Cluj-Napoca, Romania \\ Anissa Chaibi, IPAG Business School, France \\ Christophe Rault, Toulouse Business School, France
}

\begin{abstract}
This paper contributes to the existing literature by investigating how recent developments in stochastic dominance can be implemented to better understand the statistical characteristic of distributions associated with traded financial assets. In particular, we assess the impact of a shock which occurs in the evolution of a time series on the investors preferences based on data from European developed and emerging stock markets. We show that stochastic dominance tools form a useful tool in risk aversion analysis and asset allocation.
\end{abstract}

Keywords: Stochastic Dominance; Utility Function; Risk Aversion; Empirical Distributions

\section{INTRODUCTION}

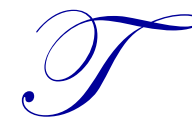

here are many applications of stochastic dominance concepts. Some of them are frequently encountered in finance and economics. Although, the stochastic dominance was applied in the early development of this concept in economics and agricultural economy for various (random) variables (McCarl, 1989), recent studies also propose these concepts as a valuable tool for choosing among distribution for various assets including also the financial ones like those described in Davidson (2006).

In this study, we contribute to the literature of stochastic dominance by studying the effect of generalized first and second order stochastic dominance changes on returns distribution of financial time series. Precisely, we consider the principal stock indexes from European countries. We show that constant relative risk aversion plays an important role in explaining the attitude for assets but the later is affected by the existing imbalances between developed and emerged markets. In the same time, we show how our measures related to stochastic dominance are affected by the financial crisis. We consider that the crisis, which affected firstly the financial sector, has extended its impact over all sectors. Therefore, our study focused on the main stock index for each analyzed country in order to capture the effect of crisis in more economic sectors and not only from the banking or financial sector. Since a stock index is considered to be a global measure of a country's economy, a behavior of investors related to this index is considered to be a good prospective of the investor to almost the entire economy from the country where this stock index is representative.

In recent years, staring with 2007, many companies' profits are on a decrease trend, which also has an impact on investors willingness for buying these types of stocks. However, the stock market is still the focus of several investors and speculators. The turbulences from past years have influenced the structure of volatility and the traditional risk measures did not capture all the features of these stock's price evolutions. Hence the implemented measure for stochastic dominance is considered to be an interesting tool for a good analysis of the crisis impact on investors' preferences and decisions.

The rest of the paper is organized as it follows: The first part of this paper presents an overview on the existing work related to stochastic dominance and illustrates its main theoretical principles. The second part deals 
with a practical example meant to stress the advantages of this concept. In the end, a summary of results is presented and some conclusions are pointed out.

\section{LITERATURE REVIEW}

The first contribution regarding the optimal behaviour of risk averse following stochastic dominance changes in returns distribution was realized by Rothschild and Stiglitz $(1970,1971)$. The research was continued by Meyer and Orminston (1985), Dionne and Gollier (1986), and Eeckhoudt et al. (1996), but these studies were not able to analyze the effects of first and second-degree stochastic dominance on optimal financial portfolio with more than two assets.

McCarl and Bessler (1989) tried to identify the bounds for a priori specification of risk aversion coefficient and found that "strongly risk averse" range might not be too high. In another paper, McCarl (1990) realized an empirical examination on risk aversion coefficients by using generalized stochastic dominance. His results show that non-dominance regions are composed of smaller dominance regions, respectively that incorporation of wealth doesn't affect generalized stochastic dominance preference interval results. Levy (1992) discussed in his paper the first, second, and third - degree stochastic dominance rules regarding portfolios with and without the riskless asset, nonlinear utility theory, arbitrage, random variables, respectively the relationship between stochastic dominance rules and risk definition.

Dachraoui and Dionne (2001) analyzed the effect of generalized first and second degree stochastic dominance on an optimal financial portfolio with two risky assets and a risk free asset. They found that deterioration in first and second order stochastic dominance will reduce the weight of risk free asset in the optimal fund.

Linton et al. (2001) realized a procedure to estimate the critical values of Kolmogorov-Smirnov test of stochastic dominance arbitrary order in a general prospect case. The study was made on daily returns of Dow Jones Industrials and S\&P 500 stock returns during the period 1988-2000. They obtained, in their research, the asymptotic distribution of mentioned test for stochastic dominance of various type, respectively they demonstrated that their consistency allows generic dependence of prospects and non independent and identical distributed observations.

Barrett and Donald (2002) used Kolmogorov-Smirnov type tests to analyze an arbitrary degree of stochastic dominance. The analyzed data was the family income from Canada during 1978-1986. They also used a lot of simulation and bootstrap methods in order to conduct inference for different degrees of stochastic dominance beyond the first order. Dentcheva and Ruszczyński (2003) tested a new model of portfolio optimization which involved stochastic dominance constraints on a portfolio of 719 real-world assets return. The study used weekly returns during the period 1990-2002.

Davidson (2006) realized a theoretical paper. He analyzed the relationship between stochastic dominance and welfare, stochastic dominance and poverty, respectively stochastic dominance and inequality. Another theoretical study was made by Gollier and Kimball (2008). They studied the comparison of risks-especially results related to stochastic dominance. The study results show that new stochastic orders can be derived from standard non-contingents.

Cowell and Victoria-Feser (2007) proposed to estimate the Lorenz curves (a fundamental tool for stochastic dominance), respectively to combine empirical estimations with a robust estimation of the upper tail distribution by using the household disposable incomes from United Kingdom during 1981 year.

Bazen and Moyes (2011) tried to measure the elitism by using stochastic dominance in their paper. The study was focused on two fields. The first field answered to the question of what is the most effective way for increase the welfare of a society. This study was focused on the comparison of 17 countries using income date. In the second field, they measured the scientific performance of academics and institutions in terms of research. This application was made on the journals from the Journal Economic Literature by departments. Their results show that the more unequal and the more efficient is the distribution, the higher it is ranked. 


\section{METHODOLOGY}

An important application of previous concepts could be found in signal processing and especially in time series analysis (Wolfstetter, 1996). The presented application is related to investment decisions in stock markets from Europe. If the first and the second order of dominance may not reveal very important information for investors, higher order of dominance, which are using more complex methods are leading to better results.

Sometimes, the variance can give different results in risk measurement. Thus, the variance of a random variable could be the same for other random variable with a different distribution. In order to illustrate this idea, one can use two random variables having the same expectation and the same variance. In this case, it is very hard for an investor who has a higher aversion at risk to choose between these two distributions (variables). Thus, one can state that a variable is more risky than another, based on the following definition:

One can say that $F_{w}$ is less riskier than $F_{w^{\prime}}$ if the two distribution have the same expectation. - i.e., $E_{w}=E_{w^{\prime}}$ and all the investor who are not willing to take risk will prefer $w$ instead of $w^{\prime}-$ i.e., $E U(w) \geq E U\left(w^{\prime}\right)$ for any utility function having the property: $U^{\prime}>0$ et $U^{\prime \prime}>0$.

A second good definition (mean preserving spread) of risk is stating that $F_{w}$ could be generated from $F_{w^{\prime}}$ by a mean preserving spread if it is possible to pass from $F_{w}$ to $F_{w^{\prime}}$ by using an expansion (i.e., a spread) - from the group of probabilities for values close to centre to higher values, without modifying the mean. In other words, the gliding of the probabilities thicken the distribution queues and emaciate it's centre.

The transformation of distribution into another one by using a mean preserving spread imply that the two obtained graphics for repartition functions (i.e., cumulative distribution functions) are crossing one each other in a single point and this point is where their associated expectations(means) are equals.

Consequently, the next definition is concerning the concept of higher risk:

$F_{w}$ is less riskier than $F_{w^{\prime}}$ if:

$A(\beta)=\int_{\alpha}^{\beta}\left[F_{w^{\prime}}(t)-F_{w}(t)\right] d t=0-$ mean preserving spread;

$A(x)=\int_{\alpha}^{x}\left[F_{w^{\prime}}(t)-F_{w}(t)\right] d t \geq 0$ for any $x \in[\alpha, \beta]-$ growth of the expansion

It can be noted that in this definition, the first condition will imply that $E_{w}=E_{w^{\prime}}$. Finally: $A(\alpha)=0$ accordingly with its definition. Since it is obvious that is possible to use this results in order to prove the validity of first statement, then it is possible to have the desired equivalence.

The mean preserving spread criteria is implying a second order stochastic dominance if the case where the distribution have the same mean. For a random variable for which the stochastic dominance degree was calculated, it is also possible to associate with the existence of this certain order of stochastic dominance, the mean preserving spread. Consequently, one "higher risk" measure for the risk of an asset is the definition of risk based on noise.

It is possible to state that $w^{\prime}$ is riskier than $w$, if a random variable $\varepsilon$ with a zero conditional mean exists (i.e., $E\{\varepsilon \mid w\}=0$ ) so that $w^{\prime}=w+\varepsilon$. Thus, the variable $w^{\prime}$ is built up by adding the noise $\varepsilon$ to $w$. Therefore, it is easy to observe that $F_{w^{\prime}}$ is obtained from $F_{w^{\prime}}$ by using the expansion of a mean preserving spread. 
Finally, by knowing that the following expression states true: $f(w, \varepsilon)=f_{\varepsilon}(\varepsilon) f(w \mid \varepsilon)=f_{\varepsilon}(w) f(w \mid \varepsilon)$, we can write the next statement that holds true: $E\{E\{\varepsilon \mid w\}\}=\int_{w}[\underbrace{\left.\int_{\varepsilon}^{\varepsilon f(\varepsilon \mid w) d \varepsilon}\right]}_{=0} f w(w) d w=0$

Related to the definition of risk based on the noise definition, the asset $w$ is less riskier from the noise point of view in regards with $w^{\prime}$, if all the investors who manifest a strongly risk aversion will prefer $w$ better than $w^{\prime}$.

Accordingly the expectation of a variance is exploited in this study by ranking it for various markets for both periods, before the crisis and after the financial crisis from 2007/2008. The next section presents the data used for these analyses.

\section{DATA}

In order to test different aspects of stock exchange indices following the effects of the last global crisis, we use daily closing data of twelve indices from European developed and emerging stock markets: AEX (Netherlands), ATX (Austria), CAC40 (France), DAX (Germany), FTSE 100 (England), and SWISS (Switzerland) from developed markets; and BET (Romania), BUX (Hungary), SAX (Slovakia), PX (Czech Republic), and WIG (Poland) from emerging markets. Analyzed time begins with the first day listing of each index (January 3, 1983 - AEX; January 7, 1986 - ATX; September 19, 1997 - BET; January 2, 1991 - BUX; July 9, 1987 - CAC40; December 10, 1987 DAX; June 30, 1978 - FTSE; September 7, 1993 - PX; July 3, 1995 - SAX; October 20, 2000 - SOFIX; July 1, 1988 - SWISS; April 16, 1991 - WIG) and ends on June 27, 2012. All closing values of the indices are collected from Datastream database, respectively are denominated in local currency.

We analyzed the stochastic dominance before and after the appearance of the last global financial crisis. Thus, we divided the analyzed period in two sub samples. The breaking point was considered the fist day of decreasing index after the registered high value of the index: July 17, 2007 (AEX); July 10, 2007 (ATX); August 25, 2007 (BET); July 23, 2007 (BUX); June 4, 2007 (CAC40); July 16, 2007 (DAX); June 18, 2007 (FTSE); October 15, 2007 (PX); March 26, 2008 (SAX); October 22, 2007 (SOFIX); June 4, 2007 (SWISS); June 7, 2007 (WIG).

The main descriptive statistics of daily return series corresponding to the twelve analyzed indices for the period before the current crisis are presented in Table 1. We can observe that the mean return series are positive in all examined markets, to the extremes being placed Bulgaria (18.83\%) and France (3.72\%). A first argument that returns do not follow a normal distribution law is given by the Kurtosis coefficient (has higher values of 3), which means that the distribution is leptokurtic, which is much less sharp than the normal distribution, and by the asymmetry coefficient (Skeweness) which is different from zero indicating a left asymmetry (except Romania, Czech Republic, Bulgaria, and Poland); i.e., the left tail is longer. The second argument that the distribution of daily stock market returns do not follow a normal distribution law is given by the value of Jarque-Bera test.

Table 1: Descriptive Statistics of Return Series Before Financial Crisis

\begin{tabular}{|c|c|c|c|c|c|c|c|c|c|c|}
\hline Ticker & $\begin{array}{l}\text { No. } \\
\text { obs. }\end{array}$ & Mean & Median & Maximum & Minimum & Std. Dev. & Skewness & Kurtosis & $\begin{array}{c}\text { Jarque- } \\
\text { Bera } \\
\end{array}$ & Prob. \\
\hline AEX & 6187 & 0.048943 & 0.080758 & 11.83107 & -11.9961 & 1.284827 & -0.13302 & 11.10912 & 16970.05 & 0 \\
\hline ATX & 5329 & 0.044393 & 0.05278 & 7.911205 & -8.91689 & 1.154836 & -0.27856 & 9.729295 & 10123.72 & 0 \\
\hline BET & 2465 & 0.108336 & 0.057598 & 15.69238 & -11.2208 & 1.755135 & 0.321717 & 10.55365 & 5902.809 & 0 \\
\hline BUX & 4151 & 0.095121 & 0.057979 & 14.58622 & -16.5006 & 1.611432 & -0.44733 & 15.19399 & 25856.18 & 0 \\
\hline CAC40 & 5004 & 0.037212 & 0.04382 & 8.573194 & -9.64065 & 1.31876 & -0.16043 & 7.379753 & 4020.962 & 0 \\
\hline DAX & 4922 & 0.043612 & 0.083527 & 7.844188 & -12.8094 & 1.392126 & -0.25349 & 8.367117 & 5960.333 & 0 \\
\hline FTSE & 6027 & 0.050997 & 0.068032 & 12.93418 & -16.6025 & 1.147646 & -0.29291 & 23.4088 & 104684.5 & 0 \\
\hline PX & 3375 & 0.060835 & 0.049628 & 16.63805 & -7.2872 & 1.371216 & 1.388451 & 19.73581 & 40471.69 & 0 \\
\hline SAX & 3084 & 0.040347 & 0.005262 & 10.04704 & -10.849 & 1.285807 & -0.25322 & 9.758351 & 5902.236 & 0 \\
\hline SOFIX & 1722 & 0.188334 & 0.104497 & 23.45832 & -18.86 & 1.835663 & 0.559474 & 39.10659 & 93629.28 & 0 \\
\hline SWISS & 4764 & 0.044965 & 0.073438 & 7.747976 & -10.517 & 1.125032 & -0.3399 & 9.837661 & 9372.322 & 0 \\
\hline WIG & 3587 & 0.138303 & 0.081947 & 15.93164 & -10.7245 & 2.12413 & 0.201971 & 8.747389 & 4961.367 & 0 \\
\hline
\end{tabular}


Return series became negative after the appearance of financial crisis for all analyzed stock markets (Table 2). Kurtosis coefficients remain higher than the value of three, therefore the distributions are leptokurtic, and these do not follow the normal law (according to Jarque-Bera test). Before the implementation process, only distributions of BET and PX return indices have a right asymmetry, and for the other indices the distribution remains have a left elongated tail. As regards the asymmetry coefficients, they present positive values for seven return series (AEX, ATX, BUX, CAC40, DAX, FTSE, and SWISS). Thus, these indices have right asymmetry.

Table 2: Descriptive Statistics of Return Series After Financial Crisis

\begin{tabular}{|c|c|c|c|c|c|c|c|c|c|c|}
\hline Ticker & $\begin{array}{l}\text { No. } \\
\text { obs. }\end{array}$ & Mean & Median & Maximum & Minimum & Std. Dev. & Skewness & Kurtosis & $\begin{array}{c}\text { Jarque- } \\
\text { Bera }\end{array}$ & Prob. \\
\hline AEX & 1268 & -0.03475 & -0.02135 & 10.54834 & -9.14481 & 1.745994 & 0.10335 & 9.085968 & 1959.152 & 0 \\
\hline ATX & 1229 & -0.05576 & -0.02030 & 12.77341 & -9.74456 & 2.1275 & 0.087064 & 6.619163 & 672.2968 & 0 \\
\hline BET & 1215 & -0.04517 & 0.014495 & 11.14274 & -12.2929 & 2.076829 & -0.27369 & 8.215685 & 1392.34 & 0 \\
\hline BUX & 1230 & -0.02537 & -0.03338 & 14.08544 & -11.8816 & 2.060513 & 0.319914 & 8.663506 & 1664.84 & 0 \\
\hline CAC40 & 1299 & -0.03718 & -0.01277 & 11.17617 & -9.03681 & 1.800336 & 0.315593 & 8.100978 & 1429.895 & 0 \\
\hline DAX & 1261 & -0.02025 & 0.040623 & 11.40189 & -7.22950 & 1.72665 & 0.323315 & 8.617033 & 1679.715 & 0 \\
\hline FTSE & 1269 & -0.00313 & 0.007804 & 9.838771 & -8.84927 & 1.557533 & 0.100992 & 8.456245 & 1576.278 & 0 \\
\hline $\mathrm{PX}$ & 1183 & -0.04584 & -0.06456 & 13.1609 & -14.9435 & 1.945186 & -0.04032 & 12.97836 & 4908.175 & 0 \\
\hline SAX & 1062 & -0.07527 & 0 & 12.61476 & -13.7655 & 1.330421 & -1.39784 & 28.47525 & 29063.59 & 0 \\
\hline SOFIX & 1146 & -0.15067 & -0.05648 & 7.564913 & -10.7385 & 1.614512 & -0.70771 & 9.878899 & 2355.158 & 0 \\
\hline SWISS & 1273 & -0.02609 & 0.014663 & 11.39097 & -7.78810 & 1.411577 & 0.292486 & 9.626728 & 2347.397 & 0 \\
\hline WIG & 1021 & -0.01475 & 0.000599 & 6.272617 & -7.95459 & 1.537889 & -0.18153 & 5.752936 & 328.0168 & 0 \\
\hline
\end{tabular}

\section{DISCUSSION OF RESULTS}

There are many important aspects in regard to the obtained results, which we want to point out in order to emphasis the relevance of the presented method. Before going into a deeper analysis of how some financial events have been affected the investor preferences for a certain country represented by its main stock index, some remarks are necessary.

The stochastic dominance analysis is a concept that strongly relays on distribution of analyzed assets (prospects). The way in which this distribution is constructed has an important influence on the experimental results and some financial decisions. It is possible to build the distribution of the prices (value of indexes, in our case) or the distribution of the returns. If the price for a specific day (e.g., let's say day $t$ ) is defined as $P_{t}$ then the return is defined as: $R_{t}=\ln \left(P_{t} / P_{t-1}\right)$. Apparently, there could be specific no interest to use one or another way of computing the distribution. Since the returns are presenting a higher interest in the stock market world and also due to the fact that the distribution of returns is close to a normal distribution (which could lead to a better econometrical modeling), we chose to use this representation as a basis for constructing the repartition functions for each of the analyzed index.

As can be seen from the Figure 1, it is rather difficult to estimate what order of dominance exist between the returns variables. Thus, other approaches like high order of stochastic dominance can be used. Figure 1 shows how difficult is to make a distinction which distribution would be preferable by an investor when one has to choose between some financial assets. 


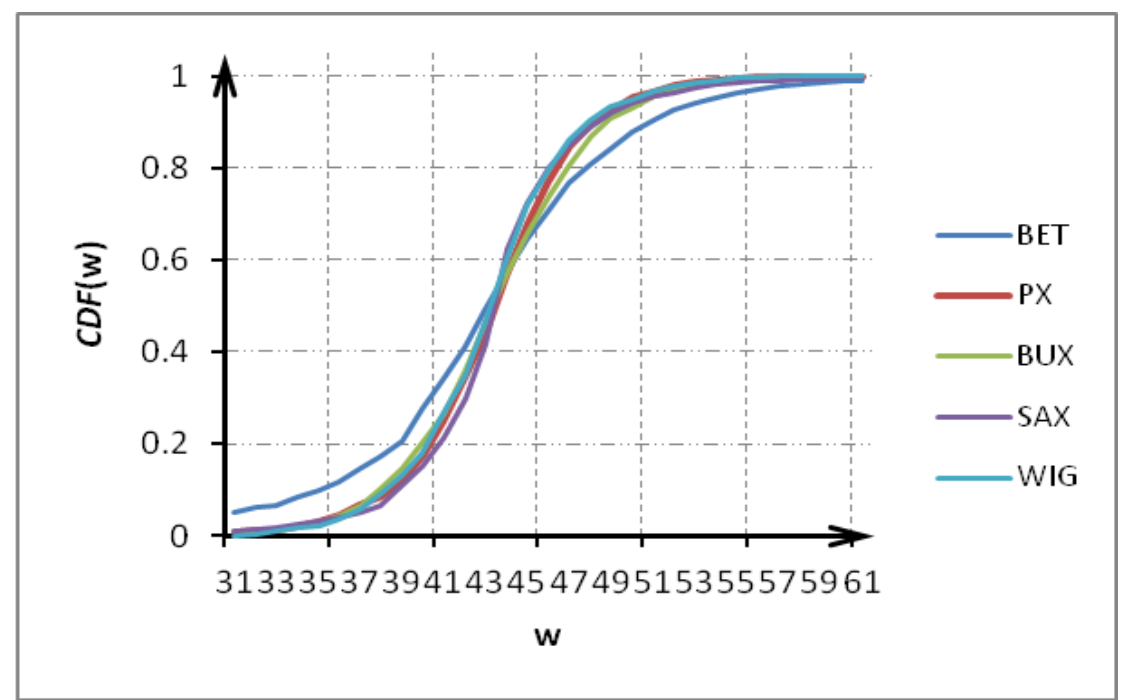

Figure 1: Differences of Distribution When Using Stochastic Dominance Techniques Source: The author analysis of available data

Figure 1 presents the cumulative distribution functions (CDF) for the return of stock indexes from six countries. Figure 1 is part of the complete representation of the entire repartition functions. The represented indexes are from the Eastern Europe countries and they have from some point of view similar characteristics, which are reflecting also in their distributions too.

The results of applying the described methodology can be presented in various ways. We chose to represent the measure of difference between distributions in two tables where each line/column is computed the dominance between assets by using the higher order of dominance measure., the first one is revealing the results before the start of the crisis and the second one on the preferences of stock market players for certain indexes after the beginning of the crisis. The tables are on the triangular form since if one asset dominates the other one and the other one is dominated and there is no need to present again the same results but the sign of value changed.

Table 3: Generalized Stochastic Dominance for Principal Stock Indexes From Europe Before the Start of Financial Crisis

\begin{tabular}{|l|c|c|c|c|c|c|c|c|}
\hline & BET & CAC & DAX & FT & PX & SOFX & SWISS & WIG \\
\hline BET & 0 & & & & & & & \\
\hline CAC & 3.849 & 0 & & & & & & \\
\hline DAX & 3.764 & 0.7924 & 0 & & & & & \\
\hline FT100 & 4.623 & 1.4361 & 1.564 & 0 & & & & \\
\hline PX & 1.639 & -0.024 & -0.004 & -0.003 & 0 & & & \\
\hline SOFX & -0.147 & -0.187 & -0.148 & -0.115 & -0.12 & 0 & & \\
\hline SWISS & 3.617 & -0.002 & 0.657 & 0.0162 & 2.494 & 5.349 & 0 & \\
\hline WIG & 2.075 & -0.023 & -0.023 & 0.0285 & 0.541 & 4.443 & -0.368 & 0 \\
\hline
\end{tabular}

The results presented in Table 3 are reflecting the stochastic dominance of higher order in the preferences of willing-to-take-risk investors during the period before the financial crisis. In this case the time frame for each index is very different since there are countries for one can get data for very long periods since for others the period is relatively short. Independently to length of the period, the distributions have the same size and therefore the results are referring the so-called "period before the 2007/2008 crisis." On the other side, it is possible to make a top of dominance, but one has to take into account that if the value of computed measure for a certain asset is different compared with that obtained in case of other asset, the only which is taken into account is the sign. Unfortunately, this study is not covering also the topic related to size of bins when computing the normalized distribution, which could lead to interesting conclusions to a refined result.

After the crisis, the situation changed in the sense that there are other distributions which became dominant, compared with those before the crisis. A similar table with Table 3 is presented below in order to emphasis the effect 
of crises and the imbalance in stock indexes' preferences changes in the emerged and developed European countries (See Table 4).

Table 4: Generalized Stochastic Dominance for Principal Stock Indexes From Europe After the Beginning of Crisis

\begin{tabular}{|l|c|c|c|c|c|c|c|c|}
\hline & BET & CAC & DAX & FT & PX & SOFX & SWISS & WIG \\
\hline BET & 0 & & & & & & \\
\hline CAC & 2.171 & 0 & & & & & \\
\hline DAX & 1.856 & 0.007 & 0 & & & & \\
\hline FT100 & -1.922 & 0.009 & 0.789 & 0 & & & \\
\hline PX & 1.536 & -0.071 & -0.00 & -0.071 & 0 & & & \\
\hline SOFX & -1.044 & 0.001 & 0.079 & 0.119 & 0.605 & 0 & & \\
\hline SWISS & 2.044 & 0.233 & 0.079 & 0.189 & 0.465 & -0.004 & 0 & \\
\hline WIG & -0.319 & 0.009 & 0.397 & -0.007 & 0.080 & -0.002 & 0.395 & 0 \\
\hline
\end{tabular}

It is interesting that there are situations when we cannot state exactly if there exists completely dominance between two distributions of the indexes for both periods. There are situations when the change in sign indicate also a change in preferences of investors.

It could be seen that in countries from Eastern Europe the changes in preferences are related to higher volatility, which characterizes these markets. In this cases the structure of volatility that has a strong randomly character has lead to an influence of the crisis over the preferences of investor with high aversion at risks.

\section{CONCLUSIONS AND FURTHER RESEARCH}

Stochastic dominance is the measure of uncertainty, which apparently involves simple approaches, but for a more complex analysis more advanced mathematical and statistical tools is required. The approach used in this paper, is a good tool which offers an interesting view about how stochastic dominance tools can be used in investment decisions. The results are relevant from risk perspective when trading on financial markets.

The presented approach could be enhanced in the sense that there could be a constructed portfolio composed of different assets and the analysis should be performed in order to optimize the constructed portfolios.

Our results are showing several approaches and aspects related to stochastic processes used in financial modeling. There are two main categories of remarks related of this study. One category is referring to general aspects when doing variance estimations for first and second order stochastic dominances methods and the other one is related to particularities of the presented results.

The attractive features of these methods based on higher order of stochastic dominances are related to the fact that volatility updating structure permits analytical solutions to be generated for standard asset prices and thus the model allows a fast calibration to given market data.

The findings of our study demonstrate that stock market investors can use different tools for analysis and ranking the risk of their investments and the fact that the financial crisis, which started in 2007, had a different impact on stock markets across Europe. The changes in preferences for certain stock index are reflected by the change in value of stochastic dominance measure like high order of stochastic dominance.

\section{AUTHOR INFORMATION}

Maria-Lenuța Ciupac-Ulici, Commercial Academy Foundation, Romania. E-mail: ulici_maria@yahoo.com

Mircea-Cristian Gherman, Technical University of Cluj-Napoca, Romania. E-mail: broker_hedger@yahoo.com

Anissa Chaibi, IPAG Business School, France. E-mail: anissa.chaibi@ipag.fr (Corresponding author)

Christophe Rault, Toulouse Business School, France. 


\section{REFERENCES}

1. Barrett, G., \& Donald S. G. (2002). Consistent tests for stochastic dominance. Department of Economics University of New South Wales, Sydney, NSW, 2052 and, Department of Economics.

2. Bazen, C., \& Moyes, T. (2011). Risk preferences in multi-period consumption models, the equity premium puzzle, and habit formation utility. The Journal of Monetary Economics, 8, 1497-1515.

3. Cowell, F., Feser, F., \& Pia, M. (2007). Robust stochastic dominance: A semi-parametric approach. The Journal of Economic Inequality, 5(1), 21-37.

4. Davidson, R. (2006). Stochastic dominance by, GREQAM, Centre de la Vieille Charite, Department of Economics, McGill University, Montreal, Quebec, Canada, H3A 2 T7.

5. Dentcheva, D., \& Ruszczynski, A. (2003). Portfolio optimization with stochastic dominance constraints.

6. Dachraoui, K., \& Dionne, G. (2001). Stochastic dominance and optimal portfolio. (Working Paper 01-01).

7. Dionne, G., \& Gollier, C. (1996). A model of comparative statistics for changes in stochastics returns with dependent risky assets. Journal of Risk and Uncertainty, 13, 147-162.

8. $\quad$ Eeckhoudt, L., Gollier, C., \& Schlesinger, H. (1996). Changes in background risk and risk taking behaviour. Econometrica, 64, 683-689.

9. Gollier, C. (2008). New methods in the classical economics of uncertainty: Comparing risks 1. Toulouse School of Economics, Miles S. KIMBALL, University of Michigan.

10. Levy, H. (1992). Stochastic dominance and expeted utility: Survey and analysis. Management Science, 38(4), 555-593.

11. Linton, O., Maasoumi, E., \& Whang, Y.-J. (2003). Consistent testing for stochastic dominance under general sampling schemes (pp. 409-423).

12. Meyer, J., \& Orminston, M. B. (1985). Strong increases in risk and their comparative statics. International Economic Review, 26, 425-437.

13. McCarl, A. B, \& Besseler, D. A. (1989). Estimating an upper bound on the Pratt risk aversion coefficient wehen the utility function is unknown. Australian Journal of Agricultural Economics, 33(1).

14. McCarl, A. B. (1990). Generalized stochastic dominance: An empirical examination. Southern Journal of Agricultural Economics.

15. Rothschild, M., \& Stiglitz, J. (1970). Increasing risk: A definition. Journal of Economic Theory, 2, 225243. 